\title{
Diskursgemeinschaften in der digitalen Transformation. Begriffsbestimmungen, Zugänge und Ziele
}

\author{
Discourse Communities in Digital Transformation. \\ Definitions, Approaches and Purposes
}

\begin{abstract}
ZUSAMMENFASSUNG
In diesem Beitrag wird im Kontext der sprachbezogenen Diskursforschung das Konzept der Diskursgemeinschaft in der digitalen Transformation diskutiert. Unter einer systemtheoretischen Deutung von Digitalisierung wird das Verständnis von 'Diskursgemeinschaft' vertieft. Diese neue Sichtweise erlaubt es, mit Hilfe von Bezügen zur Grundlagenforschung und zur angewandten Diskurslinguistik analoge und digitale Ansätze zu Diskursgemeinschaften analytisch zu trennen. Der Artikel zeigt grundlegende Unterschiede in der Modellierung des Konzepts 'Diskursgemeinschaft' auf der Basis theoretischer und methodologischer Positionen auf.

Schlüsselwörter: Diskursanalyse, Diskursgemeinschaft, digitale Transformation, Digitalisierungstheorie
\end{abstract}

\begin{abstract}
This article discusses the concept of discourse community in the context of language-related discourse research in the digital transformation. In a system-theoretical interpretation of digitalization, the understanding of 'discourse community' can become more profound. This new insight allows to analytically separate analog approaches and digital approaches to discourse communities with references to basic research and applied discourse linguistics. The article points out fundamental differences in the modeling of the concept of 'discourse community' based on theoretical and methodological positions.
\end{abstract}

Keywords: discourse analysis, discourse community, digital transformation, theory of digitalization

Philipp Dreesen, Institute of Language Competence, Zürcher Hochschule für Angewandte Wissenschaften, Theaterstr. 17, 8400 Winterthur, Phone: 00410589344255, philipp.dreesen@zhaw.ch, https://orcid.org/0000-0001-5291-2798

Julia Krasselt, Institute of Language Competence, Zürcher Hochschule für Angewandte Wissenschaften, Theaterstr. 17, 8400 Winterthur, julia.krasselt@zhaw.ch, https://orcid.org/0000-0003-1060-2657

Peter Stücheli-Herlach, Institut für Angewandte Medienwissenschaften, Zürcher Hochschule für Angewandte Wissenschaften, Theaterstr.15c,8400 Winterthur, peter.stuecheli@zhaw.ch, https://orcid. org/0000-0002-3560-7182 


\section{Diskursgemeinschaft in digitaler Perspektive}

Der Artikel leuchtet im Kontext der sprachbezogenen Diskursforschung ${ }^{1}$ das Konzept der Diskursgemeinschaft in der digitalen Transformation aus. Hierfür wird eine systemtheoretische Deutung von Digitalisierung genutzt, die deren gesamtgesellschaftliche Funktion betont, und dadurch den Blick für das Verständnis von Diskursgemeinschaft schärfen kann (Kap. 2). Diese Schärfung erlaubt es, mit Bezügen zur Grundlagenforschung und zur angewandten Diskurslinguistik analoge Zugänge (Kap. 3) und digitale Zugänge (Kap. 4) zu Diskursgemeinschaften analytisch zu trennen. Ziel ist es, anhand von theoretischen und methodischen Positionen exemplarisch grundsätzliche Unterschiede in der Modellierung und Bewertung der Kategorie Diskursgemeinschaft aufzuzeigen (Kap. 5).

\section{Gemeinschaften und statistische Gruppen der digitalen Gesellschaft}

Die digitale Transformation der Gesellschaft erfasst neben den täglich beobachtbaren Bereichen etwa des Verkehrs (z.B. Navigationsgeräte im Auto) oder der Werbung (z.B. personalisierte Werbung) auch die Wissenschaft. Neben der Untersuchung digitaler Transformationen in verschiedenen Bereichen wird die wissenschaftliche Reflexion über die Veränderung eigener Theorien und Methoden damit zu einem dringenden Erfordernis. Diese Selbstreflexion berücksichtigt idealerweise, dass Digitalität zwar eine Eigenschaft der Untersuchungsgegenstände ist (z.B. öffentliche Diskurse in sozialen Netzwerken) jedoch auch den eigenen „Denkstil“ (Fleck, 1980) mit Untersuchungsmethoden und Techniken erfasst (für den sich derzeit die Bezeichnung Digital Humanities eingebürgert). Darüber hinaus umfasst eine solche Selbstreflexion auch mögliche Effekte wissenschaftlicher Forschung auf die digitale Transformation der Gesellschaft selbst. Diese Dimensionen der wissenschaftlichen Selbstreflexion sind auch für die Beschäftigung mit Diskursgemeinschaften von zentraler Bedeutung.

Hinsichtlich der Digitalität des linguistischen Untersuchungsgegenstands Diskursgemeinschaft hilft es beispielsweise, sich zu vergegenwärtigen, in welchen medialisierten Kommunikationsgattungen wir die Ausbildung von ,Gemeinschaft erwarten bzw. überhaupt erwarten können. Entsprechend werden wir aufmerksam auf Microblogging-Communities, auf administrierte Themen-Gruppen in sozialen Netzwerken oder auf Diskussionsverläufe in Web-Foren. Hinsichtlich der methodischen Bearbeitung mit digitalen Mitteln kann man sich vorstellen, eine grosse Menge von Web-Kommentaren mittels Sentiment-Analyse (vgl. Liu, 2010) in Stimmungskategorien zu unterteilen oder beispielsweise Praktiken der Adressierung, Kommentierung und Vernetzung auf Basis der entsprechenden digitalen Operatoren

Wir sprechen im Folgenden von sprachbezogener Diskursforschung und Diskursanalyse, wenn wir nicht nur linguistische Diskursanalysen meinen, und von Diskurslinguistik, wenn wir die linguistischen Ansätze meinen. 
in den Primärdaten (wie @- oder \#-Zeichen) zu untersuchen (vgl. Krasselt, Dreesen, \& Stücheli-Herlach, im Druck). Je nach Gegenstand und Bearbeitungsmethode werden unterschiedliche Aspekte hinsichtlich des Zusammenhangs zwischen der Digitalität der Kommunikation und der Emergenz von Gemeinschaft wichtig. In beiden Fällen drängt sich die Frage auf, wie das Digitale und das Gemeinschaftliche miteinander verknüpft sind, d.h. ob und wie sie sich gegenseitig bedingen.

Der Soziologe Armin Nassehi greift diese Frage auf, indem er gerade nicht auf die technisch-medialen Infrastrukturen von Messenger Diensten und Community-Plattformen eingeht. Vielmehr umreisst er - wenn auch noch recht abstrakt - die offensichtlichen Folgen für Kommunikation und Gemeinschaft wie folgt:

Vielleicht erzeugt Big Data so etwas wie Kollektivität - aber letztlich nur in Form von collected collectivities. Big Data erzeugt keine sozialen Gruppen, sondern statistische Gruppen. Soziale Gruppen sind auch im Internet analoge Phänomene, also sichtbar, deutlich adressierbar, identitätsstiftend, an natürlicher Sprache und Alltagspraktiken orientiert. Big Data macht aus analogen Anwendern digitale Phänomene. Big Data digitalisiert die Spuren analoger Praktiken - Bewegungsprofile auf Straßen und im Netz, Kaufverhalten, Gesundheitsdaten, Freizeitverhalten, Teilnahme an social networks etc. - in der Weise, dass zum einen Daten rekombiniert werden können, die gar nicht für die Rekombination gesammelt wurden. Zum anderen entstehen dadurch statistische Gruppen, die in der analogen Welt so gar nicht vorkommen - etwa potenzielle Käufer bestimmter Produkte, Verdächtige in Rasterfahndungen oder gesundheits- und kreditbezogene Risikogruppen [Hervorhebung original] (Nassehi, 2019, S. 302)².

Nassehi weist auf einen grundlegenden Unterschied hin, wenn er die Gruppenkohäsion (das Erleben) von der Kombinatorik (der Erzeugung) abhebt. Für das diskursanalytische Forschen bedeutet diese Unterscheidung, die digitalen Zugänge von den analogen Zugängen zu Diskursgemeinschaften getrennt betrachten zu können und auch getrennt betrachten zu müssen.

Um zu beschreiben, was mit digitalen Zugängen zu Diskursgemeinschaften gemeint ist, muss zunächst der Begriff der Digitalität klarer umrissen werden. Dies ist notwendig, um der sprachbezogenen Diskursforschung ein breiteres Spektrum zu eröffnen, als es die Analyse transtextueller Phänomene in digitalen Medien mit digitalen Methoden darstellen würde. Eine stärker selbstreflektierende Position setzt eine Theorie der digitalen Transformation der Gesellschaft und ihrer Kommunikation voraus. Diese muss sich zunächst von den konkreten, technisch-medial-kommerziellen Erscheinungsformen dieser Transformation lösen und die systemische Eigengesetzlichkeit digitaler Kommunikationssysteme erfassen können (Vorarbeiten dazu hinsichtlich global integrierter digitaler Netzwerke bei van Dijk 2012, S. 6-14; 37-48). Die Selbstbezüglichkeit von Netzwerk-Systemen der Gesellschaft ist Zeichen ihrer Globalität (Luhmann 1998, S. 145-171) und damit

2 Definitorische Abgrenzungen zwischen Big Data und großen digitalen Textkorpora könnten vorgenommen werden, sind für die Argumentation an dieser Stelle aber nicht erforderlich. 
auch ihrer reflexiven Modernität (Beck, Giddens, \& Lash, 2014). In den algorithmischen Schleifen der Nachfrage-Steigerung, der Datensammlung und selbstlernenden Analyseprozessen, die ausser ihrer Vernetzungsleistung nichts mehr repräsentieren (Luhmann, 1998, S. 151), erkennt sich diese Gesellschaft wieder. Ist die Selbstbezüglichkeit der Gesellschaft ein Merkmal der Moderne (reflexive Moderne), so sind die permanent steigenden Nachfragen, Datensammlungen und Analyseoptimierungen der digitalen Transformation ihr vorläufiger Endpunkt, in der die Gesellschaft sich als Gesellschaft erkennt:

Der Siegeszug der digitalen, also zählenden, Daten rekombinierenden Selbstbeobachtung von auf den ersten Blick unsichtbaren Regelmäßigkeiten, Mustern und Clustern ist womöglich der stärkste empirische Beweis dafür, dass es so etwas wie eine Gesellschaft, eine soziale Ordnung gibt, die dem Verhalten der Individuen vorgeordnet ist [Hervorhebung original] (Nassehi, 2019, S. 50).

Mit Nassehis Betonung, dass der Beweis für unsere Vorstellung von Gesellschaft als Strukturierung individuellen Handelns in digital transformierter Form vorliege, können weitergehende diskurstheoretische Überlegungen angestellt werden. Offenkundig ist, dass die digitale Selbstbezüglichkeit zu einer starken Nachfrage nach datenanalytischer Beobachtungen des Selbst und seiner Umwelt führt (vgl. Dreesen \& Stücheli-Herlach, 2019, S. 131-132). Das bedeutet - und hier wird der bisherige Makroblick fokussierter -, dass nicht die Gesellschaft als solche selbstbeobachtend wirkt, sondern dass dies konkrete Akteure in sozialen Systemen tun, die einen jeweils spezifischen Blick auf für sie relevante Akteurskonstellationen und Diskurspraktiken tun.

Um im Kontrast zu digitalen die analogen Zugänge zur Diskursgemeinschaft nachvollziehen zu können, ist es notwendig, sich auf Nassehis Konzeption einzulassen. So liegt ein analoger Zugang vor, wenn man beispielsweise die Praktiken des Likens und des Folgens in einer Diskursgemeinschaft untersucht. Zwar gibt es digitale Bedingungen und Effekte solcher Praktiken (etwa die Möglichkeit des aktuellen Netzwerkzugangs, der digitalen Adressierung oder der algorithmischen Vorschläge), doch entstammt der Wunsch nach bestätigenden Rückmeldungen, das Bedürfnis des Beobachtens und Kontakthaltens eindeutig den analogen Zugängen (s.u.). Zu ergänzen ist dieses Bild - Nassehis Differenzierung folgend - allerdings um die Meta-Ebene der algorithmisch gesteuerten Diskurse in sozialen Netzwerken. Diese generieren selbst entsprechende Angebote zur Gemeinschaftsbildung, etwa durch Einladungen (Triff Gleichgesinnte!) und Versprechungen (Finde Deine Freunde!) und die Erzeugung entsprechend rückkoppelnder Effekte (Filterblasen, Echokammern). Allerdings kann auch hinter solchen Praktiken eine soziale Motivation angenommen werden, so der Wunsch nach Zugehörigkeitsgefühlen, nach Austausch oder aber das Ziel von Mobbing.

Hiervon ausgehend werden im Folgenden die zwei skizzierten Zugänge für die 
sprachbezogene Diskursanalyse vertieft und konkretisiert:

Kap. 3 Analoge Zugänge: Wie lässt sich die Diskursgemeinschaft erlebende Innenperspektive von Akteuren systematisch beschreiben?

Kap. 4 Digitale Zugänge: Wie lassen sich anhand diskursanalytischer Sprachdaten Diskursgemeinschaft erzeugende Muster digitalisierter Kommunikation systematisch ermitteln?

Die Abgrenzung beider Zugänge erlaubt es, das Konzept der Diskursgemeinschaft unter den Bedingungen der digitalen Transformation zu schärfen.

\section{Analoge Zugänge zur Diskursgemeinschaft}

Zur Schärfung des Konzepts der Diskursgemeinschaft sind, den analogen Zugängen folgend, drei Aspekte wichtig.

(I) In der „Diskurslinguistik nach Foucault“ wird mit dem Konzept ,Diskursgemeinschaft‘ vor allem auf ein abstrakt bleibendes, in der Regel einsprachiges, begrenztes soziales Gefüge der Produktion, Distribution und Rezeption des sprachlich gebundenen Wissens verwiesen (vgl. etwa Spitzmüller \& Warnke, 2011, S. 181-182). Kriterium für eine solche Diskursgemeinschaft ist eine stets unterstellte Gemeinsamkeit der Diskursakteure. So schreibt Roth (2018, S. 369), es sei für den Diskurs

\footnotetext{
die Identifikation der an ihm beteiligten Kommunikationspartner eben gerade nicht möglich, da Diskursbeteiligte alle sind, die von der Gültigkeit des in ihm konstituierten Wissens betroffen sind. Dabei spielt es anders als beim Gespräch vom theoretischen Gesichtspunkt her keine Rolle, ob diese Mitglieder der Diskursgemeinschaft jemals (im Sinne des Sprecher-Hörers mündlichlokaler Interaktion) Produzent von Diskursbeiträgen waren oder auch nur sein konnten oder wer intendierter Adressat dieser Diskursbeiträge war.
}

Eine abschließende Begrenzung und Bestimmung der Kommunikationspartner ist damit unmöglich und auch nicht sinnvoll für die Bestimmung von „Diskursgemeinschaft“, (Roth, 2018, S. 369). Die Eingrenzung des Begriffs erfolgt vielmehr über die Reduktion auf (in der Regel) eine Sprache oder eine Diskursebene wie bspw. die Mediendiskurse (Habscheid, 2009, S. 80). Mit Verweis u.a. auf Rampton (2000) wird bei Spitzmüller und Warnke (2011, S. 181) auf die - diesem Konzept immanenten - Gefahren der sprachideologischen Konstruktion von Diskursgemeinschaften hingewiesen, die in konkreten Forschungsvorhaben Wirkung entfalten können und deshalb in der Selbstreflexion der Diskursforschung keinesfalls ignoriert werden dürfen ${ }^{3}$.

\footnotetext{
3 Insofern ist auch zu überlegen, wie eine Abgrenzung zum Konzept der ,imagined communities“ (Anderson, 1983) besteht - eine Überlegung, die sich auch in Bezug auf virtuelle Gemeinschaften in digitalen Räumen stellt.
} 
(II) In einem Forschungsfeld der Angewandten Linguistik, den Applied Linguistics (vgl. Davies \& Elder, 2008) mit Schwerpunkt auf Sprachvermittlung, ist das Konzept der discourse community von Swales (J. Swales, 1990) verbreitet: Dem Swale'schen Konzept liegt eine Charakterisierung von discourse community zugrunde, die unter anderem aus gemeinsamen Zielen, Kommunikationsformen (genres) unter den Mitgliedern und einem bestimmten Maß an Kompetenz der Mitglieder besteht. Ansätze der sprachbezogenen Diskursforschung ignorieren dieses weitaus enger gefasste Diskursgemeinschaftskonzept bisher überwiegend (für Ausnahmen vgl. Dreesen, 2018, S. 226-227; Luttenberger \& Ritt, 2017, S. 112).

(III) Die diskursanalytische Ethnografie (vgl. etwa Macgilchrist, Ott, \& Langer, 2014, S. 40) und zuletzt auch die Akteurs-Netzwerk-Theorie (vgl. z.B. Krieger \& Belliger, 2014; Knorr-Cetina, 1999) argumentieren, dass zum Verständnis von Gruppenprozessen mindestens die teilnehmende Beobachtung erforderlich ist. Eine andere Möglichkeit besteht in der transdisziplinären Forschung, in der das Verhältnis zwischen Forschenden und Beforschten insofern aufgehoben ist, als beide gemeinsam an einer Problemlösung arbeiten (für die Linguistik vgl. Perrin \& Kramsch, 2018). Dort, wo die Expertise der Praxis in wissenschaftliche Verfahren eingebettet wird, um Lösungen für komplexe Praxisfragen zu entwickeln, wird auch für die (angewandte) Diskursforschung wichtig, dass sie mit der Innenperspektive der Praxisakteure verschränkt werden kann: Beispielsweise, wenn es um die Bestimmung diskursrelevanter Akteure, Medien und (jeweils schlagwortartig definierten) Suchwörtern in Schweizer Energiediskursen geht (ausführlich dargelegt anhand anwendungsorientierter Forschungen in Stücheli-Herlach, Ehrensberger-Dow, \& Dreesen, 2018; Stücheli-Herlach, Borghoff, Schwarz, \& Bilat, 2019).

Den Zugängen (I) bis (III) ist gemeinsam, dass sie zu einem kategoriellen Problem führen können, das im Zugang (II) von Swales (1990, S. 25) als „Café Owner Problem“ bezeichnet wird. Swales (1990) definiert zwar: „A discourse community has mechanisms of intercommunication among its members" (S. 25). Er muss dann aber diskutieren, inwiefern dies eine notwendige Bedingung für eine discourse community ist:

A, B and C may be lighthouse keepers on their lonely rocks, or missionaries on their separate jungles, or neglected consular officials in their rotting outposts. In all these cases, although A, $\mathrm{B}$ and $\mathrm{C}$ may never interact, they all have lines of communication back to base, and presumably acquired discourse community membership as a key element in their initial training (Swales, 1990, S. 25).

Es wäre absurd anzunehmen, dass die angeführte Gemeinsamkeit von A, B und $\mathrm{C}$ nicht zu einer kategoriellen Vorstellung des Gemeinsamen führen würde (eben etwa als Café-Besitzer); anderseits ist die reine Feststellung von Gemeinsamkeit keine hinreichende Bedingung dessen, was Swales als discourse commu- 
nity bezeichnet. Somit wird am Café Owner Problem ein Unterschied zwischen den beiden Zugängen (I) und (II) deutlich: Für Swales ist ein Mechanismus (z.B. ein fachliches Netzwerk, eine gemeinsame Zeitschrift) zur unmittelbaren Kommunikation gemeinschaftskonstituierend, auch wenn durch diese Beschränkung Aspekte eines umfangreicheren Gemeinsamen unberücksichtigt bleiben; hingegen besteht für die Diskurslinguistik eine Diskursgemeinschaft aus der unterstellten gemeinsamen Rezeptionsbedingung von Betroffenen, unabhängig von unmittelbarer Kommunikation ${ }^{4}$.

Die (III) transdisziplinäre Forschung versucht, die in (I) unterstellten oder festgestellten Gemeinsamkeiten in Diskursen mit den in (II) als relevant angesehenen tatsächlichen und potenziellen Interaktionen zu verbinden.

\section{Digitale Zugänge zur Diskursgemeinschaft}

Digitale Zugänge gehen davon aus, dass mittels digitaler Datenkombinationen neue Diskursgemeinschaften erzeugt werden können und dass diese ,statistischen Gruppen" durch die Analyse der Datenkombinationen auch identifizierbar sind. Für die sprachbezogene Diskursforschung bedeutet dies, die datengeleitete Identifikation text- und akteursübergreifender Muster (vgl. grundlegend Bubenhofer, 2009) des Sprachgebrauchs unter der Voraussetzung ihrer Digitalität zu nutzen, um das Konzept der Diskursgemeinschaft unter veränderten Bedingungen zu konkretisieren. Um dieses Verfahren nachvollziehbar darzustellen, werden im Folgenden Prämissen und Analyseschritte aus dem Forschungsprozess angewandter Diskurslinguistik (vgl. Dreesen \& Stücheli-Herlach, 2019) genutzt.

Auch die Modellierung digitalisierter öffentlicher Diskurse beginnt zwingend mit analog orientierten Vorüberlegungen zu thematischen Aspekten wie beispielsweise Politikfeldern, denen unterstellt wird, dass sie Gemeinsamkeit zwischen Akteuren durch einen übergreifenden Bezug auf gesellschaftliche Prozesse schaffen (Münch, 2016, S. 90-95; am Fallbeispiel der Energiediskurse vgl. StücheliHerlach et. al. 2018, S. 29-32). Die diskursive Vernetzung dieser Akteure kann nun aber nicht auf einer Ebene angenommen und untersucht werden, die sich durch Themen einerseits und autorisierte Textquellen anderseits, bestimmt wäre. Zwar suggerieren WWW-Suchmaschinen genau auf diese Weise eine leichte Verfügbarkeit von Sprachdaten. Die Reflexion auf die Digitalität der Diskurse und ihre Analyse erzwingt und ermöglicht angemessenere Methoden, um unter Diskursakteuren (a) deren sprachliche Gemeinsamkeiten (b) beschreiben zu können.

Ad (a) Zur Bestimmung von Akteuren beispielsweise von nationalen Energie-

4 Unbestritten ist, dass der Hauptunterschied darin besteht, dass im Diskursgemeinschaftskonzept der Diskurslinguistik nach Foucault kein zielgerichtetes koordiniertes Handeln vorgesehen ist, während bei Swales (1990) das gemeinsame Ziel gerade Sinn und Zweck der Gemeinschaft ausmacht. 
diskursen sind verschiedene Typen sprachlich-digitaler Adressierung zu unterscheiden. Der Akteur kann zunächst als Äußerungsinstanz (Goffman, 1979) eines ins Korpus gelangenden Textes erfasst werden; entsprechend wird er in Systemen der Annotation von Korpusdaten als Quelle erfasst. Angesichts der grossen Zahl möglicher Datenquellen für ein Korpus müssen Kategorien und Eingrenzungen für spezifische Fragestellungen etwa im Sinne eines Politikfeldes vorgenommen werden; eine bestimmte Zahl von Akteursquellen wird damit zu einer Gruppe von „untersuchten Akteuren“. In der im Korpus so erfassten Diskursproduktion werden darüber hinaus bestimmte Akteure (und auch solche, die nicht zu den untersuchten gehören) genannt, in diesem Fall reden wir von ,referenzierten Akteuren“. Der Unterschied zwischen den untersuchten und den referenzierten Akteuren ergibt sich dabei nicht nur aus der Differenz zwischen Annotationen (in denen die untersuchten Akteure erfasst und kategorisiert werden) und den primären Sprachdaten (in denen zusätzliche referenzierte Akteure erwähnt werden). Referenzierte Akteure treten zudem auch in ganz unterschiedlicher sprachlicher Gestalt auf, welche wiederum auf ihre Musterhaftigkeit untersucht werden kann: Diskurse konstituieren nicht nur individuelle, organisationale oder personale Akteure (Partei X, Person Y) und soziale Gruppen (Wähler, Angestellte, Frauen), sondern auch ganze Diskursgemeinschaften als Akteure (durch Ko-Vorkommen in Texten, durch Zuschreibungen von Zielen, Eigenschaften etc.), wie bspw. die Umweltakteure, die Energieriesen in der Schweiz, die Medien, die Energiepolitik, die EU, der Energiemarkt (Dreesen, Krasselt, Runte, \& Stücheli-Herlach, im Druck).

Ad (b) Wenn der Kreis der Diskursakteure nach diesen methodischen Differenzierungen bestimmt ist, können wiederum datenzentriert die sprachlichen Gemeinsamkeiten unter diesen Akteuren analysiert werden - und dies erneut auf eine Weise, die nicht analog konzipierte Themen unterstellen muss, sondern auf Phänomene sprachlich-digitaler Vernetzung abzielt. Zwei Zugänge lassen sich analytisch trennen, bauen in der Forschung i.d.R. jedoch aufeinander auf:

(I) Eine sprachbezogene Diskursanalyse kann die Wortschätze von unterschiedlichen Diskursgemeinschaften miteinander vergleichen (Bubenhofer, Calleri, \& Dreesen, 2019, S. 219-222) und die diskurscharakteristische Lexik von Akteursgruppen bestimmen (Dreesen et al., im Druck). Darüber hinaus stehen verschiedene, aus der maschinellen Sprachverarbeitung und Computerlinguistik stammende Verfahren zur Verfügung, die in einer quantitativ und korpuszentriert arbeitenden Diskursanalyse adaptiert werden können, um beispielsweise Sprachhandlungen, diskursive Praktiken oder im Diskurs dominant oder peripher behandelte thematische Kontexte zu identifizieren. Probabilistisch arbeitende Algorithmen wie beispielsweise Topic Modeling ermöglichen es, einen Zusammenhang zwischen statistisch nachweisbaren, semantisch kohärenten Gruppen von Wörtern und ihrem Gebrauch durch einzelne Akteure herzustellen. Auf diese Weise ergeben sich Einblicke in Gemeinsamkeiten von Akteursgemeinschaften anhand von 
Mustern ihres digitalisierten Sprachgebrauchs. Eine analog begründete Ordnung von Akteuren kann damit einerseits bestätigt werden, insbesondere - und darin liegt der Wert der digitalen Zugänge - auch erweitert und hinterfragt werden, um zu neuen Gemeinschaften von Akteuren zu gelangen (Stücheli-Herlach et al., 2019, S. 22-23).

(II) Die in (I) genannten Methoden zur korpuszentrierten Identifikation von Sprachgebrauchsmustern können in einem Netzwerk resultieren. Dieses zeigt sowohl untersuchte Akteure, referenzierte Akteure und Muster des Sprachgebrauchs (beispielsweise charakteristische Lexik und thematische Kontexte, aber auch textuelles Ko-Vorkommen von Bezeichnungen für Akteure) in Form von Knoten, die immer dann miteinander verknüpft sind, wenn eine Beziehung diskurs- und korpuslinguistisch nachweisbar ist. In Anlehnung an die soziale Netzwerkanalyse, einem klassischen Paradigma der Sozial- und Politikwissenschaften (Blondel, Guillaume, Lambiotte, \& Lefebvre, 2008; Friemel, 2008; Scott \& Carrington, 2011) eröffnen solche Diskursnetzwerke eine Reihe von Analyseoptionen.

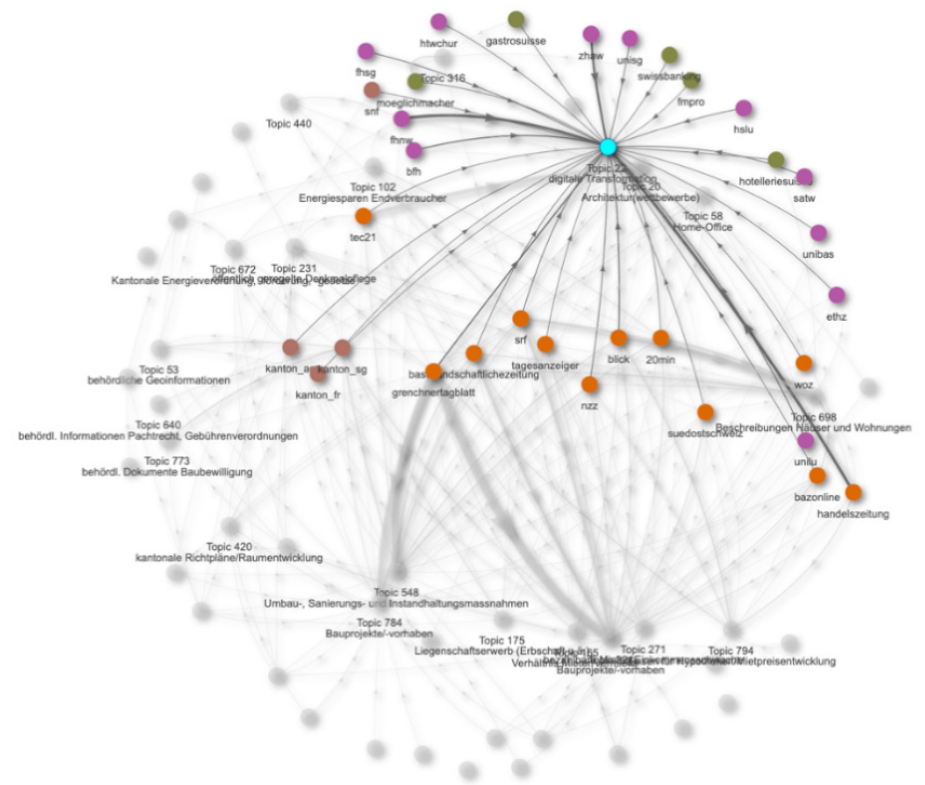

Abbildung 1: Diskursnetzwerk zur Immobilienwirtschaft in der Deutschschweiz

Eine thematische Modellierung des Diskurses mittels Topic-Modeling-Verfahren führt zunächst datengeleitet und basierend auf dem signifikanten Co-Vorkommen von Wörtern zur Identifikation von Themen, welche mittels Offener Kodierung zu Topic-Clustern im Sinne übergeordneter Themenkategorien zusammengefasst werden (z.B. Topic 22: digitale Transformation, Gebäude, Vermietung, 
Architektur). Der Gebrauch dieser thematischen Cluster durch die einzelnen, im Korpus vorkommenden Akteure (im Sinne von Textproduzenten) lässt sich unmittelbar quantifizieren, da für jeden Text die Themenzusammensetzung in Form von prozentualen Angaben bekannt ist. Sowohl Themen als auch Akteure sind im Netzwerk als Knoten dargestellt. Kommt ein Thema in den Texten eines Akteurs vor, sind die entsprechenden Knoten miteinander verbunden, wobei die Stärke der Verbindungskante genutzt werden kann, die Frequenz des Themengebrauchs durch diesen spezifischen Akteur visuell sichtbar zu machen (je stärker, desto häufiger).

Durch die Anwendung von Netzwerkalgorithmen entstehen bedeutungstragende Visualisierungen wie das Netzwerk des Diskurses zur Immobilienwirtschaft in der Deutschschweiz (vgl. Abb. 1): Knoten mit ähnlichen direkten Nachbarknoten stehen auch im Netzwerk räumlich nah beieinander. Im Falle von Diskursnetzwerken wie jenem in Abb. 1 stehen Akteure mit einem ähnlichen „Topic-Gebrauch“ näher beieinander als Akteure, die unterschiedliche Themen bespielen. Auffallend, aber nicht wenig überraschend ist so beispielsweise die Nähe von Knoten, die Schweizer Massenmedien repräsentieren (orange: z.B. SRF, NZZ, 20min, Blick, Tagesanzeiger). Von grösserem Erkenntniswert hingegen ist die Nähe von Knoten, die wichtige Schweizer Hochschulen repräsentieren (violett: ZHAW, UniSG, FHSG) und zentrale Branchenvertreter (grün: FMPRO, Gastrosuisse).

\section{Zweck digitaler Zugänge für die Diskursforschung}

Ein Zweck der digitalen Zugänge liegt beispielsweise in der Simulationen von Analyseergebnissen transdisziplinärer Diskurslinguistik (Dreesen \& StücheliHerlach, 2019, S. 150-153). Damit ist eine auf der Triangulation von Einzelergebnissen aufbauende, aggregierende Formulierung von Forschungsergebnissen mit dem Ziel gemeint, einzelne perspektivische Aspekte des Wissens über Diskurse in Reflexionsgemeinschaften diskutieren zu können. Erzeugt die Diskursforschung über ihre digitalen Zugänge „statistische Gruppen“ in Nassehis Sinne, so erzeugt sie digitales Wissen über digitalisierte Diskursgemeinschaften. Denn die so gewonnenen Ergebnisse sind selbst digitale Artefakte, welche bestimmte Merkmale digitaler Diskursgemeinschaften darstellen und so dazu dienen, die gesellschaftliche Reflexion über die digitale Transformation anzuregen und anzuleiten. Diskursanalytische Simulationen sind also nicht robuste Aussagen über moderne Diskursgemeinschaften; es sind Aussagen über die digitalen Kommunikationsbedingungen, unter denen digitale Diskursgemeinschaften in bestimmten Zeiträumen und im Kreis jeweils untersuchter Akteure entstehen.

Die Interaktionsforschung spricht von ,synthetischen Situationen“, um auf Artifizialität moderner Kommunikation hinzuweisen (Knorr Cetina, 2009). Solche synthetische Situationen emergieren aus hochgradig technisierten „skopischen Systemen“. Übertragen auf die digitallinguistische Simulation digitaler Diskursgemeinschaften heißt das: Erkenntnisse etwa über digitale Diskursnetzwerke zeigen 
nicht die Gemeinschaften, aber spezifische Möglichkeiten, wie Diskursakteure gemeinschaftsbezogen Sprache gebrauchen und damit die „skopischen“ Kommunikationssysteme des WWW verstehen und gestalten. Dies kann als Basis für eher basale oder eher angewandte Schlussfolgerungen dienen, wobei der Diskurslinguistik keine zentrale, wohl aber eine begleitende und allenfalls moderierende Funktion zukommt.

Wie gezeigt, betrifft die digitale Transformation der Gesellschaft die Wissenschaft in besonderem Maße, weil sie nicht nur neue Beschreibungen und Erklärungen liefert, sondern auch das für Forschung erforderliche Entwicklungstempo bestimmt. Für die Diskursanalyse, die sich insbesondere für die kommunikativen und epistemologischen Bedingungen von Gesellschaft interessiert, bringt dies die Notwendigkeit mit sich, diesen gesellschaftlichen Transformationsprozess wie auch die sich wandelnden Untersuchungsgegenstände und Forschungsperspektiven darauf zu reflektieren.

Im Rahmen der oben beschriebenen Bedingungen einer selbstbezüglichen Vernetzung digitaler Kommunikation in der modernen Gesellschaft bedeutet das für die Diskursforschung, dass sie ihre Vorannahmen über Diskursgemeinschaften systematisch reflektieren und in der Hypothesenbildung gezielt berücksichtigen muss. Während die digitalen Zugänge zu Mustern des Sprachgebrauchs zwar zunehmend einfacher wird (teilweise weniger Kompetenzanforderungen, schnellere, kostengünstigere und komplexere Verfahren etc.), sind die Anforderungen an die theoretisch-methodologische Reflexionsleistung mindestens gleich hoch geblieben. Diese umfassen u.a. kategorielle Fragen und deren epistemologische Auswirkungen, wie die analogen und digitalen Zugänge zur Kategorie der Diskursgemeinschaft oben gezeigt haben.

\section{References}

Anderson, B. R. O. (1983). Imagined communities: Reflections on the origin and spread of nationalism. London, New York: Verso.

Beck, U., Giddens, A., \& Lash, S. (Eds.). (2014). Reflexive Modernisierung: Eine Kontroverse. Berlin: Suhrkamp.

Blondel, V. D., Guillaume, J.-L., Lambiotte, R., \& Lefebvre, E. (2008). Fast unfolding of communities in large networks. Journal of Statistical Mechanics: Theory and Experiment, 10, 10008. DOI: 10.1088/1742-5468/2008/10/P10008.

Bubenhofer, N. (2009). Sprachgebrauchsmuster. Korpuslinguistik als Methode der Diskurs- und Kulturanalyse. Berlin, New York: De Gruyter.

Bubenhofer, N., Calleri, S., \& Dreesen, Ph. (2019). Politisierung in rechtspopulistischen Medien: Wortschatzanalyse und Word Embeddings. Osnabrücker Beiträge zur Sprachtheorie (OBST), 95, 211-241.

Davies, A., \& Elder, C. (2008). Applied Linguistics: Subject to Discipline? In A. Davies, \& C. Elder (Eds.), The Handbook of Applied Linguistics (pp. 1-15). Hoboken: Wiley-Blackwell. DOI: 10.1002/9780470757000.ch.

Dijk, J. van (2012). The Network Society. London: Sage. 
Dreesen, Ph. (2018). Diskurslinguistik und die Ethnographie des Alltags. In I. H. Warnke (Eds.), Handbuch Diskurs (pp. 265-284). De Gruyter. DOI: 10.1515/9783110296075-011.

Dreesen, Ph., Krasselt, J., Runte, M., \& Stücheli-Herlach, P. (in press). Operationalisierung der diskurslinguistischen Kategorie ,Akteur'. Triangulation und Reflexion eines drängenden Desiderats der angewandten Forschung. In M. Meiler, \& M. Siefkes (Eds.), Linguistische Methodenreflexion im Aufbruch. Beiträge zu einer aktuellen Diskussion im Schnitt-punkt von Ethnographie und Digital Humanities, Multimodalität und Mixed Methods. Berlin, New York: De Gruyter.

Dreesen, Ph., \& Stücheli-Herlach, P. (2019). Diskurslinguistik in Anwendung. Ein transdisziplinäres Forschungsdesign für korpuszentrierte Analysen zu öffentlicher Kommunikation. Zeitschrift für Diskursforschung, 7(2), 123-162.

Fleck, L. (1980). Entstehung und Entwicklung einer wissenschaftlichen Tatsache. Einführung in die Lehre vom Denkstil und Denkkollektiv. Berlin: Suhrkamp.

Friemel, T. N. (2008). Netzwerkanalytische Methoden zur Identifizierung von Kommunikationsrollen. In C. Stegbauer (Ed.), Netzwerkanalyse und Netzwerktheorie. Ein neues Paradigma in den Sozialwissenschaften (pp. 179-190). Wiesbaden: Springer.

Goffman, E. (1979). Footing. Semiotica, 25(1-2), 1-29.

Habscheid, St. (2009). Text und Diskurs. Stuttgart: W.Fink/UTB.

Knorr Cetina, K. (1999). Epistemic cultures: How the sciences make knowledge. Cambridge, MA: Harvard University Press. DOI: 10.2307/j.ctvxw3q7f.

Knorr Cetina, K. (2009). The Synthetic Situation: Interactionism for a Global World. Symbolic Interaction, 32(1), 61-87. DOI: 10.1525/si.2009.32.1.61.

Krasselt, J., Dreesen, Ph., \& Stücheli-Herlach, P. (in press). Digitalisierung des Schlagworts mittels Hashtags. Eine Fallstudie zum deutschsprachigen Klima- und energiepolitischen Diskurs. In L. Gautier, \& S. Michel (Eds.), Linguistik des Hashtags. Berlin, New York: De Gruyter.

Krieger, D. J., \& Belliger, A. (2014). Interpreting Networks: Hermeneutics, Actor-Network-Theory \& NewMedia. Bielefeld: transcript.

Liu, B. (2010). Sentiment analysis and subjectivity. In N. Indurkhya, \& F. Damerau (Eds.), Handbook of natural language processing, 2, 627-666.

Luhmann, N. (1998). Die Gesellschaft der Gesellschaft. Frankfurt am Main: Suhrkamp.

Luttenberger, J., \& Ritt, S. (2017). „Gottheit wäre mir sonst zu einfach“. Narrative Genres als Rahmen für Positionierungspraxen am Beispiel eines Computerspiel-Internetforums. wiener linguistische gazette, 8(1), 107-136.

Macgilchrist, F., Ott, M., \& Langer, A. (2014). Der praktische Vollzug von „Bologna“. Eine ethnographische Diskursanalyse. In J. Angermuller, M. Nonhoff, E. Herschinger, F. Macgilchrist, M. Reisigl, J. Wedl, ... A. Ziem (Eds.), Diskursforschung: Ein interdisziplinäres Handbuch: Vol. 2 (pp. 37-57). Bielefeld: transcript.

Münch, S. (2016). Interpretative Policy-Analyse. Eine Einführung. Wiesbaden: Springer.

Nassehi, A. (2019). Muster: Theorie der digitalen Gesellschaft. München: C.H. Beck.

Perrin, D., \& Kramsch, C. (2018). Introduction: Transdisciplinarity in applied linguistics. AILA Review, 31, 1-13. DOI: 10.1075/aila.00010.int.

Rampton, B. (2000). Speech community. In J. Verschueren, J.-O. Östman, J. Blommaert, \& C. Bulcaen (Eds.), Handbook of Pragmatics: Vol. 4 (pp. 1-34). Amsterdam: John Benjamins Publishing Company. DOI: 10.1075/hop.4.spe2.

Roth, K. S. (2018). Diskurs und Interaktion. In I. H. Warnke (Ed.), Handbuch Diskurs (pp. $363-$ 387). Berlin, Boston: De Gruyter. DOI: 10.1515/9783110296075-011.

Scott, J., \& Carrington, P. J. (Eds.). (2011). The SAGE handbook of social network analysis. London: Sage.

Spitzmüller, J., \& Warnke, I. H. (2011). Diskurslinguistik: Eine Einführung in Theorien und Methoden 
der transtextuellen Sprachanalyse. Berlin, Boston: De Gruyter. DOI: 10.1515/9783110229967. Stücheli-Herlach, P., Borghoff, B., Schwarz, N., \& Bilat, L. (2019). Antibiotikaresistenzen auf die Agenda! : Schlussbericht zur anwendungsorientierten Erforschung von Mustern der sprachlichen Verständigung im öffentlichen Diskurs über Antibiotikaresistenzen mittels digitaler Daten. ZHAW. Retrieved December 31, 2020, from https://digitalcollection.zhaw.ch/handle/11475/8772.

Stücheli-Herlach, P., Ehrensberger-Dow, M., \& Dreesen, Ph. (2018). Energiediskurse in der Schweiz. Anwendungsorientierte Erforschung eines mehrsprachigen Kommunikationsfelds mittels digitaler Daten. Working Papers in Applied Linguistics, 16. Winterthur: Zürcher Hochschule für Angewandte Wissenschaften. DOI: 10.21256/zhaw-7106. Retrieved December 12, 2020, from https://digitalcollection.zhaw.ch/handle/11475/7731.

Swales, J. (1990). Genre analysis: English in academic and research settings. Cambridge: Cambridge University Press. 